OPEN ACCESS

Edited by:

Maria Teresa Ochoa, University of Southern California, United States

Reviewed by: Lu Huang,

University of Arkansas for Medical Sciences, United States Nicola Ivan Lorè,

Division of Immunology,

Transplantation and Infectious Diseases, San Raffaele Scientific Institute (IRCCS), Italy

${ }^{*}$ Correspondence: Hongsheng Wang whs33@vip.sina.com

Specialty section: This article was submitted to Microbial Immunology, a section of the journal

Frontiers in Immunology

Received: 03 August 2021 Accepted: 14 October 2021 Published: 09 November 2021

Citation:

Ge G, Jiang $H$, Xiong J, Zhang W, Shi $Y$, Tao $C$ and Wang $H(2021)$

Progress of the Art of Macrophage Polarization and Different Subtypes in Mycobacterial Infection.

Front. Immunol. 12:752657. doi: 10.3389/fimmu.2021.752657

\section{Progress of the Art of Macrophage Polarization and Different Subtypes in Mycobacterial Infection}

\author{
Gai Ge ${ }^{1}$, Haiqin Jiang ${ }^{1}$, Jingshu Xiong ${ }^{1}$, Wenyue Zhang ${ }^{1}$, Ying Shi ${ }^{1}$, Chenyue Tao ${ }^{2}$ \\ and Hongsheng Wang ${ }^{1,3,4^{*}}$

\begin{abstract}
1 Institute of Dermatology, Chinese Academy of Medical Sciences and Peking Union Medical College, Nanjing, China, 2 Imperial College London, London, United Kingdom, ${ }^{3}$ National Center for Sexually Transmitted Disease and Leprosy Control, China Centers for Disease Control and Prevention, Nanjing, China, ${ }^{4}$ Centre for Global Health, School of Public Health, Nanjing Medical University, Nanjing, China
\end{abstract}

Mycobacteriosis, mostly resulting from Mycobacterium tuberculosis (MTb), nontuberculous mycobacteria (NTM), and Mycobacterium leprae (M. leprae), is the long-standing granulomatous disease that ravages several organs including skin, lung, and peripheral nerves, and it has a spectrum of clinical-pathologic features based on the interaction of bacilli and host immune response. Histiocytes in infectious granulomas mainly consist of infected and uninfected macrophages (M $\varphi s$ ), multinucleated giant cells (MGCs), epithelioid cells (ECs), and foam cells (FCs), which are commonly discovered in lesions in patients with mycobacteriosis. Granuloma $\mathrm{M} \varphi$ polarization or reprogramming is the crucial appearance of the host immune response to pathogen aggression, which gets a command of endocellular microbe persistence. Herein, we recapitulate the current gaps and challenges during $M \varphi$ polarization and the different subpopulations of mycobacteriosis.

Keywords: mycobacteriosis, granuloma, macrophages, multinucleated giant cells, epithelioid cells, foam cells

\section{INTRODUCTION}

Mycobacteriosis is a contagious disease ravaging the skin tissue, respiratory system, and peripheral nerves, which results from Mycobacterium tuberculosis (MTb), nontuberculous mycobacteria (NTM), and Mycobacterium leprae (M. leprae). Tuberculosis (TB), caused by the MTb complex, has plagued humanity when it has killed billions of populations over the past two centuries (1). Cutaneous TB, including $1 \%$ to $2 \%$ of all cases, is a rare clinical manifestation of MTb or M. bovis infection. Rapidly growing mycobacteria, such as M. abscessus group, M. fortuitum group, M. mucogenicum, and M. smegmatis and slow-growing mycobacteria, such as M. avium complex, M. kansasii, and M. marinum, are compose of NTM (2). NTM cutaneous infection is unwonted, and predisposing factors, such as skin injury (such as gardening and fish-related injuries, injections, and surgery) or immunosuppression make up 95\% of cases (3). Leprosy, Hansen's disease, is a remarkable public health problem, especially in countries such as Brazil, India, and Indonesia (4). Leprosy is a neglected tropical disease encountered by M. leprae or M. lepromatosis. At present, effective vaccines against infection and markers for beneficial immunity are not available $(5,6)$. The inability to eradicate the bacteria can result in infection in the immune system in a 
granuloma structure. Macrophages ( $\mathrm{M} \varphi \mathrm{s})$, primary effectors of inherited response, are considered essential pathophysiologic factors in wide-spread disease procedures involved with chronic inflammation. The heterogeneity of $\mathrm{M} \varphi s$, either due to their developmental origin or their particular activation morphologies, is becoming increasingly distinct with regard to their diverse roles within infection of microbes (7). As a central part of the innate immunity and as the paramount host of infectious granuloma pathogens, $\mathrm{M} \varphi \mathrm{s}$ have been the central focus of mycobacteriosis investigation.

\section{INFECTIOUS GRANULOMA}

Granuloma is a highly structured and organized collection of $\mathrm{M} \varphi s$, often with phenotypic switches and other immune cells recruited, including multinucleated giant cells (MGCs), epithelioid cells (ECs), and foam cells (FCs). Someone claimed a new ex vivo granuloma culture technique to study granuloma consolidation (8). Mechanistically, Cronan et al. have found that in the existence of robust interferon-gamma (IFN- $\gamma$ ) signaling immune response, confronting interleukin (IL)-4 and IL-13 signals were associated with $\mathrm{M} \varphi$ epithelial transition. IL-4/13 signaling, induced by stat6, was required for epithelioid transformation and granuloma architecture. Apart from stat6 function required in the new granuloma formation, persistent stat6 pathway was required to maintain the expression of E-cadherin and granuloma (9). MAB_4780, encoding a dehydratase, was required for intracellular $M$. abscessus growth and to avoid lysosome-mediated degradation, which compromises survival of $\triangle M A B \_4780$ in $M \varphi s$ and granuloma formation (10). In granuloma transformation, IFN- $\gamma$ and tumor necrosis factor-alpha (TNF- $\alpha$ ) were deemed to be effective regulators, whereas IL-10 was a passive effector. Intriguingly, etanercept and adalimumab, the human monoclonal anti-TNF- $\alpha$ IgG1, exacerbated M1 polarization and delayed MGC generation in granuloma (11). Magically, there are two types of granulomas in leprosy. At one pole of leprosy, the presence of MGCs and granuloma configuration in tuberculoid leprosy (TT) contributes to the containment of $M$. leprae proliferation and transmission (Figure 1A). At the other pole, lepromatous leprosy (LL) has phagocytic FCs heavily parasitized with freely multiplying intracellular M. leprae (Figure 1B) (12). Ma et al. have constructed a map via integrating single-cell RNA sequencing with spatial sequencing to identify that the primary cell types, consisting of $\mathrm{T}$ cells, M $\varphi s$, keratinocytes, endothelial cells, and fibroblasts, were described to research the cellular composition and status discrepancies between reversal reactions and LL, and LGCs are more frequent in both lesions. IL- $1 \beta$ and IFN- $\gamma$ were supposed to be important upstream effectors of the pseudo time trajectory and the activation of $\mathrm{M} \varphi \mathrm{s}$ in granulomas to product genes contributing to antimicrobial responses in human leprosy granulomas (13).

Granuloma is a leading gateway for the host immune response to microorganisms and shape immune interplays, disease progression, and degeneration (14). The granuloma is a functional paradox, for example, it contains the bacilli in a local reservoir, preventing mycobacterial dissemination to near normal tissues of the host, or to shield itself from host's immunologic killing mechanisms and antimicrobial response (15).

\section{M $\varphi$ PHENOTYPES IN MYCOBACTERIOSIS}

M $\varphi$ s show the increment of plasticity, and they can be

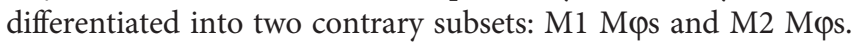
The network of molecular mediators is regulated in response to the diversity of stimulus. Binding of IFN- $\gamma$ to its cellular surface receptor, for example, induces the activation of receptor-linked JAKs, which trigger STAT1 to dimerize and translocate to the nucleus where it initiates gene transcription that skew toward M1-correlated functions such as promoted microbicidal activity and proinflammatory cytokine production (Figure 1C). By contrast, IL-4 and IL-13 activate STAT6 to promote M2 profile inhibiting these effects (Figure 1D) (16). Furthermore, Yun-Ji et al. have shown that c-JUN N-terminal kinase (JNK)-mediated M1 plasticity was important in the elimination of bacilli via p53mediated apoptosis by M $\varphi s$. Similarly, virulent MTb H37Rv infection can induce $\mathrm{M} 2 \mathrm{M} \varphi \mathrm{s}$ and in turn restrain $\mathrm{p} 53$ by the activation of mouse double minute 2 (MDM2). These consequences elucidated that M2 population decreases p53elicited cell death by MDM2 induction (17). In addition, early secreted antigenic target of $6 \mathrm{kDa}$ (ESAT6), a prerequisite step to support the formation of granuloma, was one of the effectors used by MTb to facilitate the proinflammatory $M 1 M \varphi$ at the primo-infection and then triggered the switch of M1 to M2 M $\varphi$ at a later infection phase (18). In particular, proinflammatory environment or bacterial product could perturb the classical M1 or M2 phenotypes. Bénard et al. recently showed that type I IFN hyperproduction by MTb-stimulated B cells drove an altered $\mathrm{M} \varphi$ s polarization toward a regulatory/anti-inflammatory profile, namely, M2 M $\varphi$, during TB which associated with increased MTb burden in lungs (19). Moreover, $\mathrm{M} \varphi$ polarization may augment antimicrobial response against MTb in the existence of vitamin D (20).

Furthermore, high-mobility group N2 (HMGN2) regulates antiNTM-inherited response function of $M \varphi$. In addition, HMGN2 is triggered in NTM and IFN- $\gamma$-primed M1-skewed subpopulation polarization (21). Yet, $M$. abscessus infection robustly induced p38 MAPK-dependent heme-oxygenase-1 (HO-1) induction in the THP-1 cells. HO-1 production was important for M. abscessus growth during the early stages of infection, and that the HO-1 producted bilirubin and biliverdin, perhaps through modulation of intracellular ROS levels, may be involved (22). Glycopeptidolipids limited the virulence of $M$. abscessus among $M \varphi s$ by inhibition of apoptosis and spreading of bacteria (23). In TT, the activation of the classical signal by M1 M $\varphi$ s results in the expression of TNF- $\alpha$, IFN- $\gamma$, and iNOS, which trigger the multiplication of free radicals that remove Bacillus (24). Moreover, the LL shows a superiority of M2 M $\varphi$ s that promotes the expression of IL-10, transforming growth factor- $\beta$, fibroblast growth factor- $\beta$, Arg- 1, CD206, and CD163, causing immunosuppressive response and tissue 
A

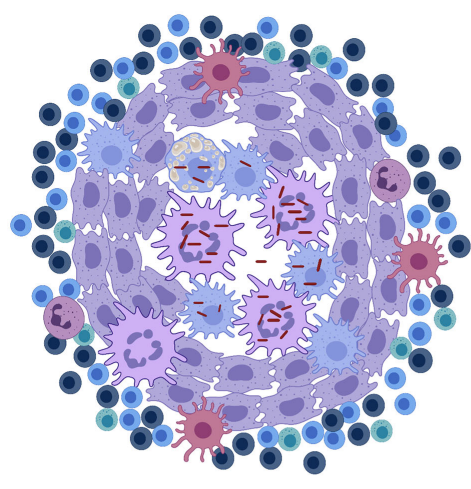

C

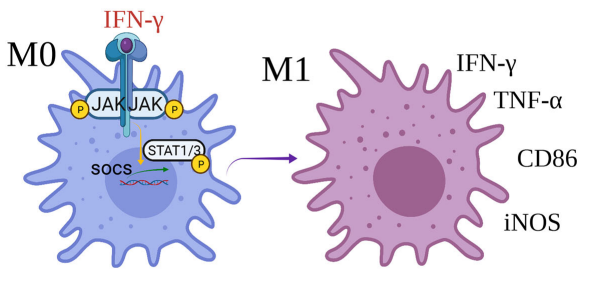

E

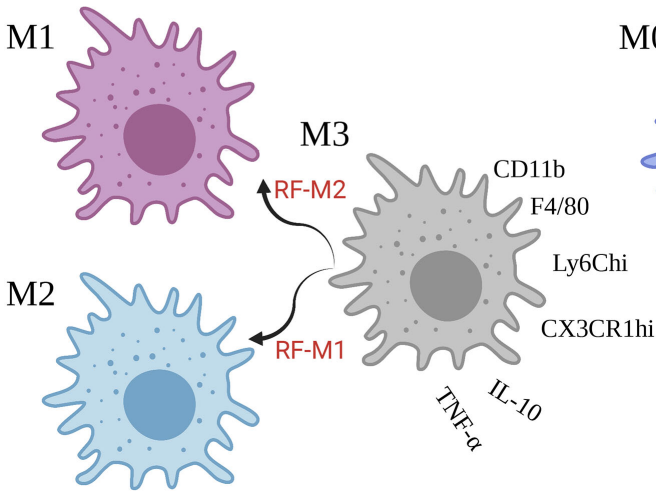

D

$\mathbf{F}$

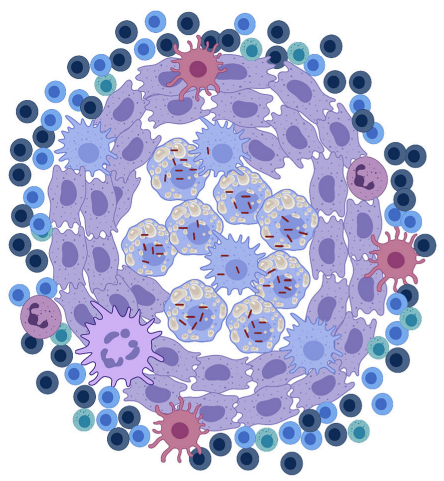

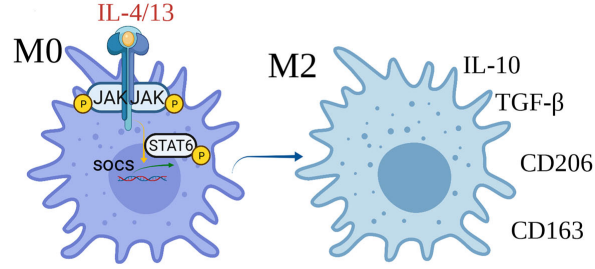

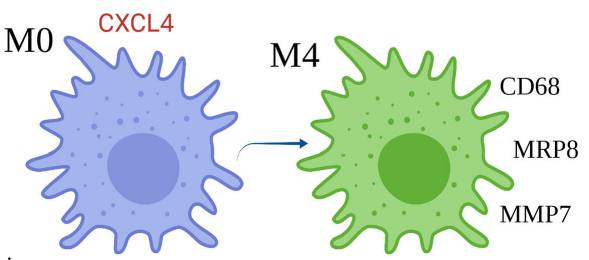

FIGURE 1 | The granuloma and macrophages subsets. (A, B) Two frameworks of mycobacterial infection granuloma. (C-F) Reprogramming of macrophages and main markers of M1, M2, M3 and M4 M $\phi$. Created with BioRender.com.

repairment $(25,26)$. $M$. leprae could then utilize infected M $\varphi$ s by two mechanisms: first, $M$. leprae-infected $M \varphi s$ preferentially activated Treg but not Th1 or cytotoxic T-cell responses; second, M. leprae-infected $\mathrm{M} \varphi$ s were effective in escaping $\mathrm{CD}^{+} \mathrm{T}$-cellprimed cytotoxicity (27).

Other than the M1 and M2 subpopulations, a M3 switch profile exists. The M3 M $\varphi$ could be divided into two subsets such as the $\mathrm{M} 1 / 2$ paradigm, which in response to a reprogramming factor M1 (RF-M1) skews toward M2 M $\varphi$, and the M2/1 dichotomy, which responding to RF-M2 favors $\mathrm{M} 1 \mathrm{M} \varphi$ (28). In murine mesothelioma microenvironment, flow cytometry disclosed that the mixture of M1 and $\mathrm{M} 2$ phenotypes $\left(\mathrm{CD} 11 \mathrm{~b}^{+} \mathrm{F} 4 / 80^{+} \mathrm{Ly}_{6 \mathrm{C}^{\text {hi }}} \mathrm{CX} 3 \mathrm{CR} 1^{\mathrm{hi}}\right)$, that was, M3 M $\varphi$, secreted IL-10 and TNF- $\alpha$. Jackaman et al. have suggested that the shifts of M1 to M2 M $\varphi$ and vice versa could occur through the M3 changing formation (Figure 1E) (29). The M3 mediator can be triggered by upregulation of M1-reprogramming signals with coinstantaneous suppression of the $\mathrm{M} 2 \mathrm{M} \varphi$ transcription factors, STAT3, STAT6, and/or SMAD3 in Ehrlich ascites carcinoma (30). Nevertheless, the role of $\mathrm{M} 3 \mathrm{M} \varphi$ in mycobacteriosis remains undetailed, and more studies are required for further investigation.

Unluckily, the part of M4 macrophages following M3 macrophages in $\mathrm{M} \varphi$ phenotypes in mycobacteriosis notably, considerable evidence for another subpopulation of $\mathrm{M} \phi$, namely, M4 M $\phi$, was frequently observed. In the presence of CXCL4, M0 M $\phi$ changed to M4 M $\phi$, expressing CD206, CD68, matrix metallo proteinase (MMP) 7, myeloid-related protein 8 (MRP8) and S100A8, producing IL-6, TNF- $\alpha$, MMP7, and MMP12 in atherosclerosis and cardiac remodeling (31-33). 
At date, de Sousa et al. have also characterized the existence of $\mathrm{M} 4 \mathrm{M} \phi$ in leprosy. Immunostaining determined that the expression of CD68, MRP8 and MMP7 was significantly higher, while IL-6 and TNF- $\alpha$ was significantly lower in the LL group compared with the TT group. The higher expression of M4 profile in LL lesions implied that the subpopulation was ineffective in the removal of bacilli, resulting in the development of multibacillary form and microbes replication (Figure 1F) (34). Further work is necessary to robustly establish this mechanism. Notwithstanding, the role of the new subset in TB and NTM is unclear.

\section{MGCS IN MYCOBACTERIOSIS}

Specific lineage of MФs, particularly MGCs containing a horseshoe-shaped ring of nuclei, contributes to the core of granulomas. Previously, cells with three nuclei and the expression of iNOS were markers for MGC transformation (35). In addition, the formation of MGC, involving cell fusion (36), was a $\mathrm{M} \varphi$-specific, evolutionarily ancient program that proceeds in response to the persistence of extrinsic and intrinsic stimuli (37). M $\varphi$ s or monocytes can be transformed into MGCs under several statuses, including cultivating with IL-4 or IL-13, GM-CSF combined with IL-4, IFN- $\gamma$ bounding with IL-3, or bacterial glycolipids. E-cadherin is a necessary player in fusion, and its production can be stimulated by the activation of STAT6 through IL-4 or IL-13 pathway, similar to epithelialization under the circumstance of schistosome granulomas (38). However, the development of polyploid MGCs involves cell autonomous affliction of Toll-like receptor-elicited DNA damage, cell autonomous cell-cycle alterations, and impairment of p53 function by the potent antimicrobial effector, namely, NO, driving mitotic defects and multinucleation $(35,39)$. Wang et al. have corroborated experimental evidence that IL-15 primes $\mathrm{M} 1 \mathrm{M} \varphi$ transformation, reprograms peripheral blood mononuclear cells in humans to transform into MGCs via direct activation of T cells and myeloid cells (40). Queval et al. have shown that out of the four infection combinations (bloodderived primary human and bovine $\mathrm{M} \phi s[\mathrm{hM} \phi$ or $\mathrm{bM} \phi$, respectively] infected with $M$. bovis and $\mathrm{MTb}$ ), bM $\phi$ infected with $M$. bovis promotes the formation of MGCs. Mechanistically, they have distinguished the functional differences between $M$. bovis and MTb host-pathogen interplay and demonstrated that MPB70 from $M$. bovis and extracellular vesicles released by $M$. bovis-infected bM $\phi$ promote $\mathrm{M} \phi$ multinucleation (41). Startlingly, local adaptive immune response, particularly programmed cell death ligand-1, fatty acid, and cholesterol metabolism could take part in containing granuloma progression in human lung TB $(42,43)$.

Unfortunately, the distinct role of MGCs in mycobacterial infection immune response remains as major gaps. MGCs may restrict mycobacterial cell-to-cell dissemination, involve in mycobacterial latency, or promote tissue destruction because of their high expression of extracellular matrix-degrading epithelioid macrophage marker molecules (EMMMs) $(38,43)$. The maturation of MGCs supplies a restrictive environment for $M$. bovis. The major lysosomal degradative signals remain functional within MGC transition. In addition, the increase of $M$. bovis in acidified compartments and correlation with LC3B in matured MGCs indicates that MGCs presented a restrictive milieu for microorganism replication (41). Nonetheless, the role of MGCs in NTM and leprosy remains an elusive issue.

\section{ECs IN MYCOBACTERIOSIS}

Microscopic analysis discloses that tightly interdigitated cell membranes are formed in zipper-like arrays to resemble epithelioid histiocytes. Nevertheless, none of the fusion molecules is strictly required to give rise to ECs, and the procedure is complicated. Epithelial differentiation can occur during days of granuloma transformation. Using the $M$. marinum-zebrafish model, Cronan et al. have found recently that granuloma $\mathrm{M} \varphi$ s undergo reprograming, which involves Ecadherin-dependent formation of fusogenic epithelial cell (44). In TB, ESAT6 plus TLR2 can activate iNOS/NO and ROS signaling to reduce the trimethylation of $\mathrm{H} 3 \mathrm{~K} 27$, thereby promoting the expression of EMMM that improved the transformation of M $\varphi$ s into ECs (45).

The EC functions are amphibolous and nebulous from being repleted with organelles and strongly phagocytic and microbicidal to being nonphagocytic cells with secretory functions, which might be adjunctive in granuloma function. Notwithstanding, some people have demonstrated by electron microscopy that the ECs in TB are "primarily biosynthetic rather than phagocytic" (46). However, ECs control the multiplication of mycobacteria at least in one experimental model. Previous dates, therefore, have elucidated that interference to E-cadherin production, a tight junction protein among ECs, enhanced the transformation of untightly structured granuloma, resulting in unrestricted $\mathrm{MTb}$ motion and leads to MTb regeneration and dissemination (47). In NTM, EC surrogates restrain $M$. avium growth and serves as APCs in vitro and in vivo. ECs were commonly seen in TT and borderline tuberculoid leprosy (BT). Inconceivably, ECs from TT granulomas exhibited the M1 phenotype $\left(\mathrm{CD} 68^{+} \mathrm{CD} 163^{-}\right)$, whereas $\mathrm{M} \varphi$ s in $\mathrm{LL}$ granulomas showed the M2 phenotype $\left(\mathrm{CD} 68^{+} \mathrm{CD} 163^{+}\right)(48)$.

\section{FCs IN MYCOBACTERIOSIS}

FCs, with deregulated lipid metabolism, are a manifestation of maladaptive responses in chronic inflammatory statuses $(49,50)$. The biogenesis of FCs varies with underlying diseases. FC biogenesis is involved in the disruption of cholesterol homeostasis and consequent endocellular accumulation of cholesteryl esters in atherosclerosis, but it is linked to triglyceride accumulation in hM $\varphi$ s infected with MTb that is elicited by TNF receptor pathway via downstream activation of 
the caspase cascade and mammalian target of rapamycin complex 1 (51). In comparison, Genoula et al. suggested that alternatively activated $\mathrm{M} \varphi s$ were loath to the accumulation of lipid droplets (LDs) via the STAT6, which facilitated the degradation of lipids. However, MTb offsets lipolysis via switching alternatively activated $\mathrm{M} \varphi$ metabolism to accumulate LDs due to the HIF-1 $\alpha$ activation (52). Similarly, the zebrafishM. marinum granuloma contained FCs and the mycobacterial ESX1 pathogenicity locus thought to elicit the morphology switch of M $\varphi$ s to FCs (53). The biogenesis of FCs in leprosy remains a challenging enigma.

Diverse, and in part controversial, we summarize the current findings in the role of FCs in mycobacteriosis. First, $\mathrm{M} \varphi$ ontology may be a major paramount factor of the inherited response in the containment of MTb infection. LDs may take part in inherent immunity against MTb by directly eliminating intracellular MTb and modulate metabolism to infection (54). Strikingly, PPAR signaling is responsible for lots of adipocyte differentiationcorrelated genes, leading to amassing of intracellular lipids to accommodate $M$. leprae parasitization in host FCs $(55,56)$. Furthermore, the formation of LD may support the host by averting access of MTb to host's fatty acids (FAs) while favoring native immune responses (54). In comparison, unlike other programs, FC formation reduced the avidity of host cell and the phagocytosis of MTb while protecting the cells from death. The protective effect is associated with enhanced inflammatory potential of FCs and cause slower proliferation of MTb. Also, the balance of TNF-a, IL-1 $\beta$, IL-6, and NF-kB innate inflammatory responses was altered in response to MTb vs. LPS in FCs compared with uninfected controls (57). Additionally, FCs triggered the formation of necrotic core by releasing triglyceriderich content into the caseum (51), resulting in progressive lung tissue destruction and pulmonary function loss in infected rabbits and marmosets and in individuals with active TB (50).

Lastly, FCs may result in TB pathogenesis by enhancing MTb persistence and drug resistance. Moreover, a lipid-rich diet rather than nutrient deprivation in caseum rewires the condition of MTb toward drug resistance (58). In addition, IL10/STAT3 axis primed FC differentiation during MTb infection, favoring pathogen persistence (59). Palma et al. have shown that controlled caloric restriction protected murine model against pulmonary $\mathrm{MTb}$ infection by decreasing bacterial load and FC proliferation to reduce lung damage and limit MTb spreading (60). Thus, the reduction of LDs in MTb-infected FCs might restrain the endocellular survival of $\mathrm{MTb}$ (61). Likewise, ultrastructural analysis of demic leprosy tissue showed colocalization between cholesterol-laden lipid bodies and $M$. leprae-containing phagosomes in FCs. The mechanisms of leprosy indicate that lipid abundance has a pathophysiological effect on the persistence of microbes in the host. The function of FCs remains the unsolved mystery of NTM.

\section{DISCUSSION}

Mycobacterium-infected disease is an infectious granuloma disease with a spectrum of clinical and pathological features. Granuloma formation and immune mechanism are primarily observed in mycobacteriosis. Different cellular immune and clinical manifestations are primed by $M \varphi$ polarization or reprogramming. Different $M \varphi$ subphenotypes may be positively correlated with the number of germs and host immune response. The increment of M2 M $\phi$ s and FCs and a low degree of MGCs are more likely to attribute to the bacillary multiplies and impaired innate immune. Conversely, the results reveal a positive correlation between high-level M1 M $\varphi s$ and MGCs, the diminution of FCs, and a limited bacterial load and immunocompetent innate immune response. Particularly, ECs are commonly seen in TT and borderline BT, FCs are mostly a commonly factor in leprosy, particularly LL. Now, we recapitulate the main findings of M $\varphi s$, MGCs, ECs, and FCs in mycobacterial infection (Table $\mathbf{1}$ ). $\mathrm{M} \varphi$ reprogramming or markers can shed light on the cell immune response in mycobacteriosis. Moreover, the mycobacterial granuloma

TABLE 1 | Main findings described in M $\varphi s$, MGCs, ECs, and FCs.

\begin{tabular}{|c|c|c|c|c|}
\hline $\begin{array}{l}\text { Cell } \\
\text { types }\end{array}$ & Stimulus & $\begin{array}{l}\text { Main cyto/chemokines and } \\
\text { enzymes }\end{array}$ & Functions/Immune responses & References \\
\hline $\mathrm{M} 1 \mathrm{M} \varphi$ & IFN- $\gamma /$ STAT1, p53, ESAT6 & $\begin{array}{l}\text { iNOS, IFN- } \gamma, \text { TNF- } \alpha, \text { CD } 86, I L-6 \text {, and } \\
\text { HMGN2 }\end{array}$ & $\begin{array}{l}\text { Microbicidal activity and proinflammatory cytokine } \\
\text { production }\end{array}$ & $(16-18,24)$ \\
\hline $\mathrm{M} 2 \mathrm{M} \varphi$ & IL-4 plus IL-13/STAT6, ESAT6, type I IFN & $\begin{array}{l}\text { Arg-1, IL-10, TGF- } \beta \text {, fibroblast growth } \\
\text { factor- } \beta \text {, CD206, CD163 }\end{array}$ & Immunosuppressive response and tissue repairment & $\begin{array}{c}(16-19,25 \\
26)\end{array}$ \\
\hline МЗ М & $\mathrm{RF}-\mathrm{M} 1 / 2$ & $\begin{array}{l}\text { IL-10, TNF- } \alpha, \text { CD11b, F4/80, Ly6C } \\
\text { CX3CR } 1^{\text {hi }}\end{array}$ & Undetailed & $(28,29)$ \\
\hline 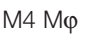 & CXCL4 & CD68, MRP8, MMP7 & Weak phagocytosis, favoring bacillus regeneration & $(35-38)$ \\
\hline MGC & $\begin{array}{l}\text { IL-4 or IL-13, GM-CSF plus IL-4, IFN- } \gamma \text { plus } \\
\text { IL-3, E-cadherin, IL-15 }\end{array}$ & iNOS, EMMMs, PD-L1 & $\begin{array}{l}\text { Inhibiting mycobacterial cell-to-cell spread or tissue } \\
\text { destruction and mycobacterial latency }\end{array}$ & $\begin{array}{c}(39,42,45- \\
47)\end{array}$ \\
\hline EC & ESAT6 plus TLR2 & $\mathrm{CD}^{+}{ }^{+} \mathrm{CD}^{163}{ }^{-}, \mathrm{CD} 68+\mathrm{CD} 163+$ & $\begin{array}{l}\text { Strongly phagocytic and microbicidal or nonphagocytic } \\
\text { cells with secretory functions }\end{array}$ & $(49,52)$ \\
\hline FC & PPAR, IL-10 & TNF-a, IL-1 $\beta, \| L-6$ & $\begin{array}{l}\text { Favoring inherited response or pathogen persistence, } \\
\text { Less-bactericidal, Less-phagocytic }\end{array}$ & $(59-61)$ \\
\hline
\end{tabular}

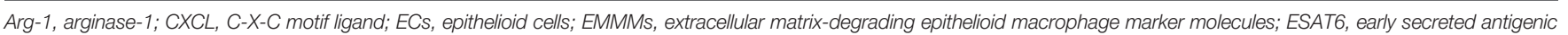
target of 6-kDa; FCs, foamy cells; HMGN2, high-mobility group N2; IFN- $\gamma$, interferon-gamma; IL, interleukin; iNOS, inducible nitric oxide synthase; IRF, Interferon regulatory factors; M $\varphi$ s, macrophages; MGCs, multinucleated giant cells; MMP, matrix metallo proteinase; MRP8, myeloid-related protein 8; PD-L1, programmed cell death ligand-1; RF-M1, reprogramming factor M1; STAT, signal transducer and activator of transcription; TGF- $\beta$, transforming growth factor beta; TNF- $\alpha$, tumor necrosis factor-alpha. 
model may delineate the development of alternative vaccines for mycobacteriosis. Accordingly, these researches prompt that M $\varphi$ s, especially M1 M $\varphi$ and LGCs represent a therapeutic target for the emergence of antibacterial immunity. Together, therapies targeting some particular cells are being studied as novel therapies for TB, leprosy, and other bacterial infections.

\section{AUTHOR CONTRIBUTIONS}

HW, HJ, JX, WZ, and YS involved in supervision. CT and GG drafted figures. GG reviewed the literature and wrote the manuscript. All authors contributed to the article and approved the submitted version.

\section{REFERENCES}

1. Reid M, Arinaminpathy N, Bloom A, Bloom BR, Boehme C, Chaisson R, et al. Building a Tuberculosis-Free World: The Lancet Commission on Tuberculosis. Lancet (2019) 393(10178):1331-84. doi: 10.1016/S0140-6736(19)30024-8

2. Franco-Paredes C, Marcos LA, Henao-Martinez AF, Rodriguez-Morales AJ, Villamil-Gomez WE, Gotuzzo E, et al. Cutaneous Mycobacterial Infections. Clin Microbiol Rev (2018) 32(1):e00069-18. doi: 10.1128/CMR.00069-18

3. Moreno G, Minocha R, Choy B, Jelfs P, Watts M, Fernandez-Penas P. Cutaneous Non-Tuberculous Mycobacteria in Western Sydney, Australia. Population Study 1996-2013. Australas J Dermatol (2018) 59(4):343-5. doi: $10.1111 /$ ajd.12783

4. Mi Z, Liu H, Zhang F. Advances in the Immunology and Genetics of Leprosy. Front Immunol (2020) 11:567. doi: 10.3389/fimmu.2020.00567

5. Cadena AM, Flynn JL, Fortune SM. The Importance of First Impressions: Early Events in Mycobacterium Tuberculosis Infection Influence Outcome. Mbio (2016) 7(2):e316-42. doi: 10.1128/mBio.00342-16

6. Huang L, Russell DG. Protective Immunity Against Tuberculosis: What Does it Look Like and How do We Find it? Curr Opin Immunol (2017) 48:44-50. doi: 10.1016/j.coi.2017.08.001

7. Khan A, Singh VK, Hunter RL, Jagannath C. Macrophage Heterogeneity and Plasticity in Tuberculosis. J Leukoc Biol (2019) 106(2):275-82. doi: 10.1002/ JLB.MR0318-095RR

8. Cronan MR, Matty MA, Rosenberg AF, Blanc L, Pyle CJ, Espenschied ST, et al. An Explant Technique for High-Resolution Imaging and Manipulation of Mycobacterial Granulomas. Nat Methods (2018) 15(12):1098-107. doi: 10.1038/s41592-018-0215-8

9. Cronan MR, Hughes EJ, Brewer WJ, Viswanathan G, Hunt EG, Singh B, et al. A Non-Canonical Type 2 Immune Response Coordinates Tuberculous Granuloma Formation and Epithelialization. Cell (2021) 184(7):1757-74. doi: 10.1016/j.cell.2021.02.046

10. Halloum I, Carrere-Kremer S, Blaise M, Viljoen A, Bernut A, Le Moigne V, et al. Deletion of a Dehydratase Important for Intracellular Growth and Cording Renders Rough Mycobacterium Abscessus Avirulent. Proc Natl Acad Sci USA (2016) 113(29):E4228-37. doi: 10.1073/pnas.1605477113

11. Mezouar S, Diarra I, Roudier J, Desnues B, Mege JL. Tumor Necrosis FactorAlpha Antagonist Interferes With the Formation of Granulomatous Multinucleated Giant Cells: New Insights Into Mycobacterium Tuberculosis Infection. Front Immunol (2019) 10:1947. doi: 10.3389/fimmu.2019.01947

12. Leal-Calvo T, Martins BL, Bertoluci DF, Rosa PS, de Camargo RM, Germano GV, et al. Large-Scale Gene Expression Signatures Reveal a Microbicidal Pattern of Activation in Mycobacterium Leprae-Infected Monocyte-Derived Macrophages With Low Multiplicity of Infection. Front Immunol (2021) 12:647832. doi: $10.3389 /$ fimmu.2021.647832

13. Ma F, Hughes TK, Teles R, Andrade PR, de Andrade SB, Plazyo O, et al. The Cellular Architecture of the Antimicrobial Response Network in Human Leprosy Granulomas. Nat Immunol (2021) 22(7):839-50. doi: 10.1038/ s41590-021-00956-8

14. Ernst JD, Cornelius A, Desvignes L, Tavs J, Norris BA. Limited Antimycobacterial Efficacy of Epitope Peptide Administration Despite

\section{FUNDING}

This study was supported by grants from the Science and Technology Planning Project of Jiangsu Province of China (BE2018619), Chinese Academy of Medical Science Innovation Fund for Medical Science (2017-I2M-B\&R-14), and the National Natural Science Foundation of China (81972950).

\section{ACKNOWLEDGMENTS}

The authors acknowledge the contributions of all the scientists in this area and apologize for failing to cite any work due to constraints of space.

Enhanced Antigen-Specific CD4 T-Cell Activation. J Infect Dis (2018) 218 (10):1653-62. doi: 10.1093/infdis/jiy142

15. Pagan AJ, Ramakrishnan L. The Formation and Function of Granulomas. Annu Rev Immunol (2018) 36:639-65. doi: 10.1146/annurev-immunol032712-100022

16. Lawrence T, Natoli G. Transcriptional Regulation of Macrophage Polarization: Enabling Diversity With Identity. Nat Rev Immunol (2011) 11 (11):750-61. doi: 10.1038/nri3088

17. Lim YJ, Lee J, Choi JA, Cho SN, Son SH, Kwon SJ, et al. M1 Macrophage Dependent-P53 Regulates the Intracellular Survival of Mycobacteria. Apoptosis (2020) 25(1-2):42-55. doi: 10.1007/s10495-019-01578-0

18. Refai A, Gritli S, Barbouche MR, Essafi M. Mycobacterium Tuberculosis Virulent Factor ESAT-6 Drives Macrophage Differentiation Toward the ProInflammatory M1 Phenotype and Subsequently Switches it to the AntiInflammatory M2 Phenotype. Front Cell Infect Microbiol (2018) 8:327. doi: $10.3389 /$ fcimb.2018.00327

19. Benard A, Sakwa I, Schierloh P, Colom A, Mercier I, Tailleux L, et al. B Cells Producing Type I IFN Modulate Macrophage Polarization in Tuberculosis. Am J Respir Crit Care Med (2018) 197(6):801-13. doi: 10.1164/rccm.201707-1475OC

20. Rao MJ, Parasa VR, Lerm M, Svensson M, Brighenti S. Polarization of Human Monocyte-Derived Cells With Vitamin D Promotes Control of Mycobacterium Tuberculosis Infection. Front Immunol (2019) 10:3157. doi: 10.3389/fimmu.2019.03157

21. Wang X, Chen S, Ren H, Chen J, Li J, Wang Y, et al. HMGN2 Regulates NonTuberculous Mycobacteria Survival via Modulation of M1 Macrophage Polarization. J Cell Mol Med (2019) 23(12):7985-98. doi: 10.1111/jcmm.14599

22. Abdalla MY, Ahmad IM, Switzer B, Britigan BE. Induction of Heme Oxygenase-1 Contributes to Survival of Mycobacterium Abscessus in Human Macrophages-Like THP-1 Cells. Redox Biol (2015) 4:328-39. doi: 10.1016/j.redox.2015.01.012

23. Whang J, Back YW, Lee KI, Fujiwara N, Paik S, Choi CH, et al. Mycobacterium Abscessus Glycopeptidolipids Inhibit Macrophage Apoptosis and Bacterial Spreading by Targeting Mitochondrial Cyclophilin D. Cell Death Dis (2017) 8(8):e3012. doi: 10.1038/cddis.2017.420

24. Simoes QJ, de Almeida FA, de Souza AT, de Miranda ASL, Nunes MI, Fuzii HT, et al. Transforming Growth Factor Beta and Apoptosis in Leprosy Skin Lesions: Possible Relationship With the Control of the Tissue Immune Response in the Mycobacterium Leprae Infection. Microbes Infect (2012) 14 (9):696-701. doi: 10.1016/j.micinf.2012.02.010

25. de Sousa JR, de Sousa RP, Aarao TL, Dias LJ, Carneiro FR, Fuzii HT, et al. In Situ Expression of M2 Macrophage Subpopulation in Leprosy Skin Lesions. Acta Trop (2016) 157:108-14. doi: 10.1016/j.actatropica.2016.01.008

26. Shapouri-Moghaddam A, Mohammadian S, Vazini H, Taghadosi M, Esmaeili SA, Mardani F, et al. Macrophage Plasticity, Polarization, and Function in Health and Disease. J Cell Physiol (2018) 233(9):6425-40. doi: 10.1002/ jcp. 26429

27. Yang D, Shui T, Miranda JW, Gilson DJ, Song Z, Chen J, et al. Mycobacterium Leprae-Infected Macrophages Preferentially Primed Regulatory T Cell Responses and was Associated With Lepromatous Leprosy. PloS Negl Trop Dis (2016) 10(1):e4335. doi: 10.1371/journal.pntd.0004335 
28. Malyshev I, Malyshev Y. Current Concept and Update of the Macrophage Plasticity Concept: Intracellular Mechanisms of Reprogramming and M3 Macrophage "Switch" Phenotype. BioMed Res Int (2015) 2015:341308. doi: $10.1155 / 2015 / 341308$

29. Jackaman C, Yeoh TL, Acuil ML, Gardner JK, Nelson DJ. Murine Mesothelioma Induces Locally-Proliferating IL-10(+) TNF-Alpha (+) CD206 (-) CX3CR1(+) M3 Macrophages That can be Selectively Depleted by Chemotherapy or Immunotherapy. Oncoimmunology (2016) 5(6): e1173299. doi: 10.1080/2162402X.2016.1173299

30. Kalish S, Lyamina S, Manukhina E, Malyshev Y, Raetskaya A, Malyshev I. M3 Macrophages Stop Division of Tumor Cells In Vitro and Extend Survival of Mice With Ehrlich Ascites Carcinoma. Med Sci Monit Basic Res (2017) 23:819. doi: $10.12659 / \mathrm{msmbr} .902285$

31. Oksala N, Seppala I, Rahikainen R, Makela KM, Raitoharju E, Illig T, et al. Synergistic Expression of Histone Deacetylase 9 and Matrix Metalloproteinase 12 in M4 Macrophages in Advanced Carotid Plaques. Eur J Vasc Endovasc Surg (2017) 53(5):632-40. doi: 10.1016/j.ejvs.2017.02.014

32. Nakai K. Multiple Roles of Macrophage in Skin. J Dermatol Sci (2021) S09231811(21):00201-2. doi: 10.1016/j.jdermsci.2021.08.008

33. Yang Y, Ma L, Wang C, Song M, Li C, Chen M, et al. Matrix Metalloproteinase-7 in Platelet-Activated Macrophages Accounts for Cardiac Remodeling in Uremic Mice. Basic Res Cardiol (2020) 115(3):30. doi: 10.1007/s00395-020-0789-z

34. de Sousa JR, Lucena NF, Sotto MN, Quaresma J. Immunohistochemical Characterization of the M4 Macrophage Population in Leprosy Skin Lesions. BMC Infect Dis (2018) 18(1):576. doi: 10.1186/s12879-018-3478-x

35. Gharun K, Senges J, Seidl M, Losslein A, Kolter J, Lohrmann F, et al. Mycobacteria Exploit Nitric Oxide-Induced Transformation of Macrophages Into Permissive Giant Cells. EMBO Rep (2017) 18(12):214459. doi: 10.15252/embr.201744121

36. Helming L, Gordon S. Molecular Mediators of Macrophage Fusion. Trends Cell Biol (2009) 19(10):514-22. doi: 10.1016/j.tcb.2009.07.005

37. Shrivastava P, Bagchi T. IL-10 Modulates In Vitro Multinucleate Giant Cell Formation in Human Tuberculosis. PloS One (2013) 8(10):e77680. doi: 10.1371/journal.pone.0077680

38. Miyamoto H, Katsuyama E, Miyauchi Y, Hoshi H, Miyamoto K, Sato Y, et al. An Essential Role for STAT6-STAT1 Protein Signaling in Promoting Macrophage Cell-Cell Fusion. J Biol Chem (2012) 287(39):32479-84. doi: 10.1074/jbc.M112.358226

39. Herrtwich L, Nanda I, Evangelou K, Nikolova T, Horn V, Sagar, et al. DNA Damage Signaling Instructs Polyploid Macrophage Fate in Granulomas. Cell (2016) 167(5):1264-80. doi: 10.1016/j.cell.2016.09.054

40. Wang H, Jiang H, Teles R, Chen Y, Wu A, Lu J, et al. Cellular, Molecular, and Immunological Characteristics of Langhans Multinucleated Giant Cells Programmed by IL-15. J Invest Dermatol (2020) 140(9):1824-36. doi: 10.1016/j.jid.2020.01.026

41. Queval CJ, Fearns A, Botella L, Smyth A, Schnettger L, Mitermite M, et al. Macrophage-Specific Responses to Human- and Animal-Adapted Tubercle Bacilli Reveal Pathogen and Host Factors Driving Multinucleated Cell Formation. PloS Pathog (2021) 17(3):e1009410. doi: 10.1371/journal.ppat.1009410

42. Abengozar-Muela M, Esparza MV, Garcia-Ros D, Vasquez CE, Echeveste JI, Idoate MA, et al. Diverse Immune Environments in Human Lung Tuberculosis Granulomas Assessed by Quantitative Multiplexed Immunofluorescence. Mod Pathol (2020) 33(12):2507-19. doi: 10.1038/s41379-020-0600-6

43. Losslein AK, Lohrmann F, Scheuermann L, Gharun K, Neuber J, Kolter J, et al. Monocyte Progenitors Give Rise to Multinucleated Giant Cells. Nat Commun (2021) 12(1):2027. doi: 10.1038/s41467-021-22103-5

44. Cronan MR, Beerman RW, Rosenberg AF, Saelens JW, Johnson MG, Oehlers $\mathrm{SH}$, et al. Macrophage Epithelial Reprogramming Underlies Mycobacterial Granuloma Formation and Promotes Infection. Immunity (2016) 45(4):86176. doi: 10.1016/j.immuni.2016.09.014

45. Lin J, Jiang Y, Liu D, Dai X, Wang M, Dai Y. Early Secreted Antigenic Target of 6-Kda of Mycobacterium Tuberculosis Induces Transition of Macrophages Into Epithelioid Macrophages by Downregulating Inos/NO-Mediated H3K27 Trimethylation in Macrophages. Mol Immunol (2020) 117:189-200. doi: 10.1016/j.molimm.2019.11.013

46. Crawford CL. The Epithelioid Cell in Tuberculosis is Secretory and Not a Macrophage. J Infect Dis (2015) 212(7):1172-3. doi: 10.1093/infdis/jiv155
47. Nathan C. Macrophages' Choice: Take it in or Keep it Out. Immunity (2016) 45(4):710-1. doi: 10.1016/j.immuni.2016.10.002

48. Fachin LR, Soares CT, Belone AF, Trombone AP, Rosa PS, Guidella CC, et al. Immunohistochemical Assessment of Cell Populations in Leprosy-Spectrum Lesions and Reactional Forms. Histol Histopathol (2017) 32(4):385-96. doi: 10.14670/HH-11-804

49. Hotamisligil GS. Foundations of Immunometabolism and Implications for Metabolic Health and Disease. Immunity (2017) 47(3):406-20. doi: 10.1016/ j.immuni.2017.08.009

50. Guerrini V, Gennaro ML. Foam Cells: One Size Doesn't Fit All. Trends Immunol (2019) 40(12):1163-79. doi: 10.1016/j.it.2019.10.002

51. Guerrini V, Prideaux B, Blanc L, Bruiners N, Arrigucci R, Singh S, et al. Storage Lipid Studies in Tuberculosis Reveal That Foam Cell Biogenesis is Disease-Specific. PloS Pathog (2018) 14(8):e1007223. doi: 10.1371/journal.ppat.1007223

52. Genoula M, Marin FJ, Maio M, Dolotowicz B, Ferreyra M, Milillo MA, et al. Fatty Acid Oxidation of Alternatively Activated Macrophages Prevents Foam Cell Formation, But Mycobacterium Tuberculosis Counteracts This Process via HIF-1alpha Activation. PloS Pathog (2020) 16(10):e1008929. doi: 10.1371/ journal.ppat.1008929

53. Johansen MD, Kasparian JA, Hortle E, Britton WJ, Purdie AC, Oehlers SH. Mycobacterium Marinum Infection Drives Foam Cell Differentiation in Zebrafish Infection Models. Dev Comp Immunol (2018) 88:169-72. doi: 10.1016/j.dci.2018.07.022

54. Laval T, Chaumont L, Demangel C. Not Too Fat to Fight: The Emerging Role of Macrophage Fatty Acid Metabolism in Immunity to Mycobacterium Tuberculosis. Immunol Rev (2021) 301(1):84-97. doi: 10.1111/imr.12952

55. Luo Y, Tanigawa K, Kawashima A, Ishido Y, Ishii N, Suzuki K. The Function of Peroxisome Proliferator-Activated Receptors PPAR-Gamma and PPAR-Delta in Mycobacterium Leprae-Induced Foam Cell Formation in Host Macrophages. PloS Negl Trop Dis (2020) 14(10):e8850. doi: 10.1371/journal.pntd.0008850

56. Arnett E, Weaver AM, Woodyard KC, Montoya MJ, Li M, Hoang KV, et al. Ppargamma Is Critical for Mycobacterium Tuberculosis Induction of Mcl-1 and Limitation of Human Macrophage Apoptosis. PloS Pathog (2018) 14(6): e1007100. doi: 10.1371/journal.ppat.1007100

57. Agarwal P, Combes TW, Shojaee-Moradie F, Fielding B, Gordon S, Mizrahi V, et al. Foam Cells Control Mycobacterium Tuberculosis Infection. Front Microbiol (2020) 11:1394. doi: 10.3389/fmicb.2020.01394

58. Sarathy JP, Dartois V. Caseum: A Niche for Mycobacterium Tuberculosis Drug-Tolerant Persisters. Clin Microbiol Rev (2020) 33(3):e00159-19. doi: 10.1128/CMR.00159-19

59. Genoula M, Marin FJ, Dupont M, Kviatcovsky D, Milillo A, Schierloh P, et al. Formation of Foamy Macrophages by Tuberculous Pleural Effusions Is Triggered by the Interleukin-10/Signal Transducer and Activator of Transcription 3 Axis Through ACAT Upregulation. Front Immunol (2018) 9:459. doi: 10.3389/fimmu.2018.00459

60. Palma C, La Rocca C, Gigantino V, Aquino G, Piccaro G, Di Silvestre D, et al. Caloric Restriction Promotes Immunometabolic Reprogramming Leading to Protection From Tuberculosis. Cell Metab (2021) 33(2):300-18. doi: 10.1016/j.cmet.2020.12.016

61. Shim D, Kim H, Shin SJ. Mycobacterium Tuberculosis Infection-Driven Foamy Macrophages and Their Implications in Tuberculosis Control as Targets for HostDirected Therapy. Front Immunol (2020) 11:910. doi: 10.3389/fimmu.2020.00910

Conflict of Interest: The authors declare that the research was conducted in the absence of any commercial or financial relationships that could be construed as a potential conflict of interest.

Publisher's Note: All claims expressed in this article are solely those of the authors and do not necessarily represent those of their affiliated organizations, or those of the publisher, the editors and the reviewers. Any product that may be evaluated in this article, or claim that may be made by its manufacturer, is not guaranteed or endorsed by the publisher.

Copyright $\odot 2021 \mathrm{Ge}$, Jiang, Xiong, Zhang, Shi, Tao and Wang. This is an open-access article distributed under the terms of the Creative Commons Attribution License (CC BY). The use, distribution or reproduction in other forums is permitted, provided the original author(s) and the copyright owner(s) are credited and that the original publication in this journal is cited, in accordance with accepted academic practice. No use, distribution or reproduction is permitted which does not comply with these terms. 